\title{
The burden of gastroenteritis in Switzerland (BUGS) study: a research proposal for a 1-year, prospective cohort study
}

\author{
Claudia Schmutz ${ }^{1,2}$ (D) and Daniel Mäusezahl $1^{1,2^{*}}$ (1)
}

\begin{abstract}
Objectives: Acute gastroenteritis (AG) is a usually self-limiting, but common disease worldwide. In Europe, incidence estimates range from 0.3-1.5 AG episodes/person-year. For Switzerland, available information on AG is restricted to notifiable foodborne diseases and findings from research studies starting at primary care level. The aims of this 1 -year, population-based prospective cohort study are to assess the incidence, burden of disease, aetiology and socio-economic impact of AG in the Swiss general population. Additionally, the prevalence of bacterial gastrointestinal pathogens and bacteria harbouring antimicrobial resistances in the asymptomatic population shall be assessed.

Results: Weekly follow-up of the cohort consisting of 3000 participants will provide incidence estimates of AG. Furthermore, information collected will be used to assess risk factors for experiencing an episode of AG, to explore determinants for help seeking, and to characterise the socio-economic impact of AG including absence from work and inability to perform daily activities. Aetiology of AG is determined by investigating stool samples from symptomatic participants. Finally, stool samples from participants collected during an asymptomatic period will be used to assess the prevalence of enterohaemorrhagic E. coli, Campylobacter spp., Salmonella spp. and Shigella spp. as well as of resistance to different antibiotics (extended-spectrum beta-lactamase-, fluoroquinolone- and carbapenemase-resistance).
\end{abstract}

Keywords: Research proposal, Cohort study, Acute gastroenteritis, Burden of disease, Incidence, Aetiology, Antibiotic resistance, Switzerland

\section{Introduction}

Acute gastroenteritis (AG), manifesting with signs and symptoms of diarrhoea, vomiting, abdominal pain or cramps, fever, dehydration, nausea and/or loss of appetite, is usually self-limiting, but leads to a considerable burden of disease, health system use and socio-economic impact. Studies in several European countries estimated the incidence of AG at 0.3-1.5 episodes per person and year [1-14]. Furthermore, it was found that a considerable $11 \%$ of patients with infectious enteritis develop post-infectious irritable bowel syndrome $[15,16]$. Incidence of AG for Switzerland is assumed to be comparable, but data is limited to notifiable pathogens reported

\footnotetext{
*Correspondence: Daniel.maeusezahl@swisstph.ch

${ }^{1}$ Swiss Tropical and Public Health Institute, Basel, Switzerland

Full list of author information is available at the end of the article
}

to the Federal Office of Public Health (FOPH) based on the Epidemics Act. Several studies from other European countries have shown that (i) only $6.4-37.8 \%$ of all AG episodes lead to consultation of a physician [1-9, 11, 12, $14]$, and (ii) $0.2-1.8 \%$ of episodes are reported to national surveillance systems $[1,11,14]$. These proportions are highly variable between countries and pathogens due to different help seeking behaviour, case management and surveillance systems [17]. From a study in the Swiss Sentinel Surveillance Network, Sentinella, we estimated that around 175,000 individuals consulted a physician due to AG in Switzerland in 2014 [18]. Around 12\% of cases were asked to submit a stool specimen and hence, could potentially-if positive-be reported to the National Notification System for Infectious Diseases (NNSID) if their sample tested positive for a notifiable pathogen and was reported by the diagnostic laboratory. However, 
the proportion of AG patients consulting a physician is currently not known for Switzerland. Consequently, inference from the above-mentioned frequencies and proportions to the incidence and burden of AG at population level is not possible.

Therefore, the present study primarily aims at measuring the incidence of acute gastroenteritis in the general population in Switzerland. Secondary objectives are to describe the burden of disease and the socio-economic impact of AG, to assess its aetiology and to investigate the frequency of selected risk exposures. Finally, the carriage rate of selected pathogenic bacteria and bacteria harbouring selected antibiotic resistances among the "healthy" (non-diarrhoeal) population in Switzerland is assessed.

\section{Main text}

\section{Study design and methodology}

A 1-year, prospective cohort study is conducted to determine the incidence of AG in the general population in Switzerland. The study will assess signs and symptoms of AG and exposure to selected risk factors (incl. antibiotic use). In case of symptoms, the help seeking behaviour of patients, inability to work or to perform usual daily activities, perceived illness experience and socio-economic consequences of the illness will be explored. Furthermore, the aetiology of AG is investigated by examining stool samples from a subsample of participants reporting gastrointestinal symptoms. Finally, the prevalence of selected bacterial gastrointestinal pathogens (enterohaemorrhagic Escherichia coli [EHEC], Campylobacter, Salmonella and Shigella) and bacteria with selected antibiotic resistances (extended-spectrum beta-lactamase [ESBL], carbapenemase, fluoroquinolone, and mobilised colistin resistance [mcr]-1) is assessed among participants during an asymptomatic period.

\section{Study setting, recruitment process and eligibility}

A representative sample of the Swiss population will be requested from the Federal Statistical Office. The cohort is recruited by postal mail. The procedure for cohort recruitment is shown in Fig. 1. Invitation letters include a study information document, an informed consent form, a contact information questionnaire (for obtaining participants' contact details and for selection of the preferred language and means of communication) and a short screening questionnaire to assess study eligibility. Eligibility criteria for participating in the study are: living in Switzerland; speaking German, French or Italian; age $\geq 14$ years; not suffering from cancer of the bowel, irritable bowel syndrome, Crohn's disease, ulcerative
BUGS cohort recruitment

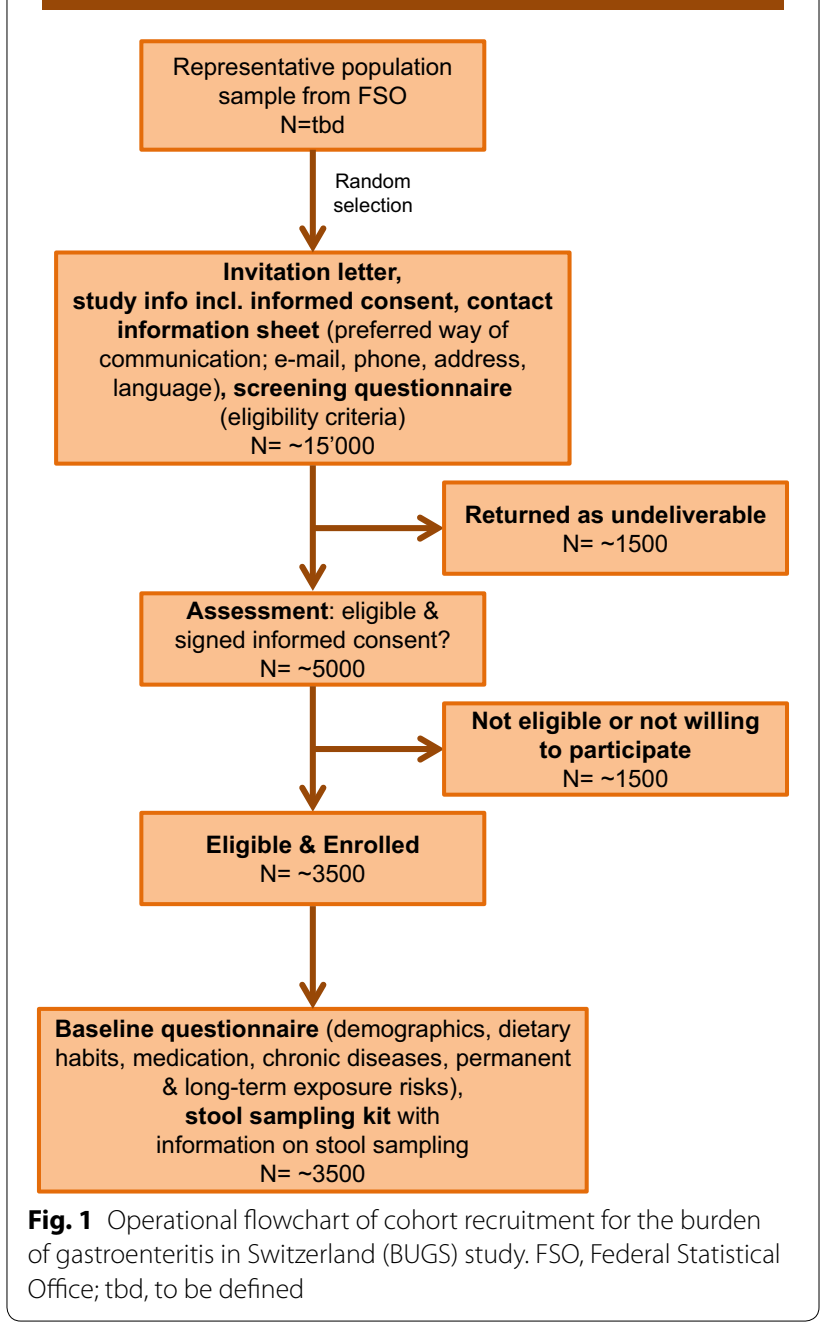

colitis, cystic fibrosis, coeliac disease or another chronic illness with symptoms of diarrhoea or vomiting.

\section{Data collection and management}

Upon return of the signed informed consent and the completed contact information and screening questionnaires, eligible study participants receive a baseline questionnaire, a stool sampling kit including an information and instruction sheet on stool sampling, and the first weekly questionnaire. The content of each questionnaire is summarised in Table 1. Participants will continuously receive the weekly questionnaire during 1 year (52 weeks; Fig. 2). Participants receive an additional questionnaire ("illness questionnaire") in case of reporting gastrointestinal signs and symptoms. Furthermore, participants are advised to immediately report occurrence of diarrhoea and/or vomiting actively to the study team by phone, 


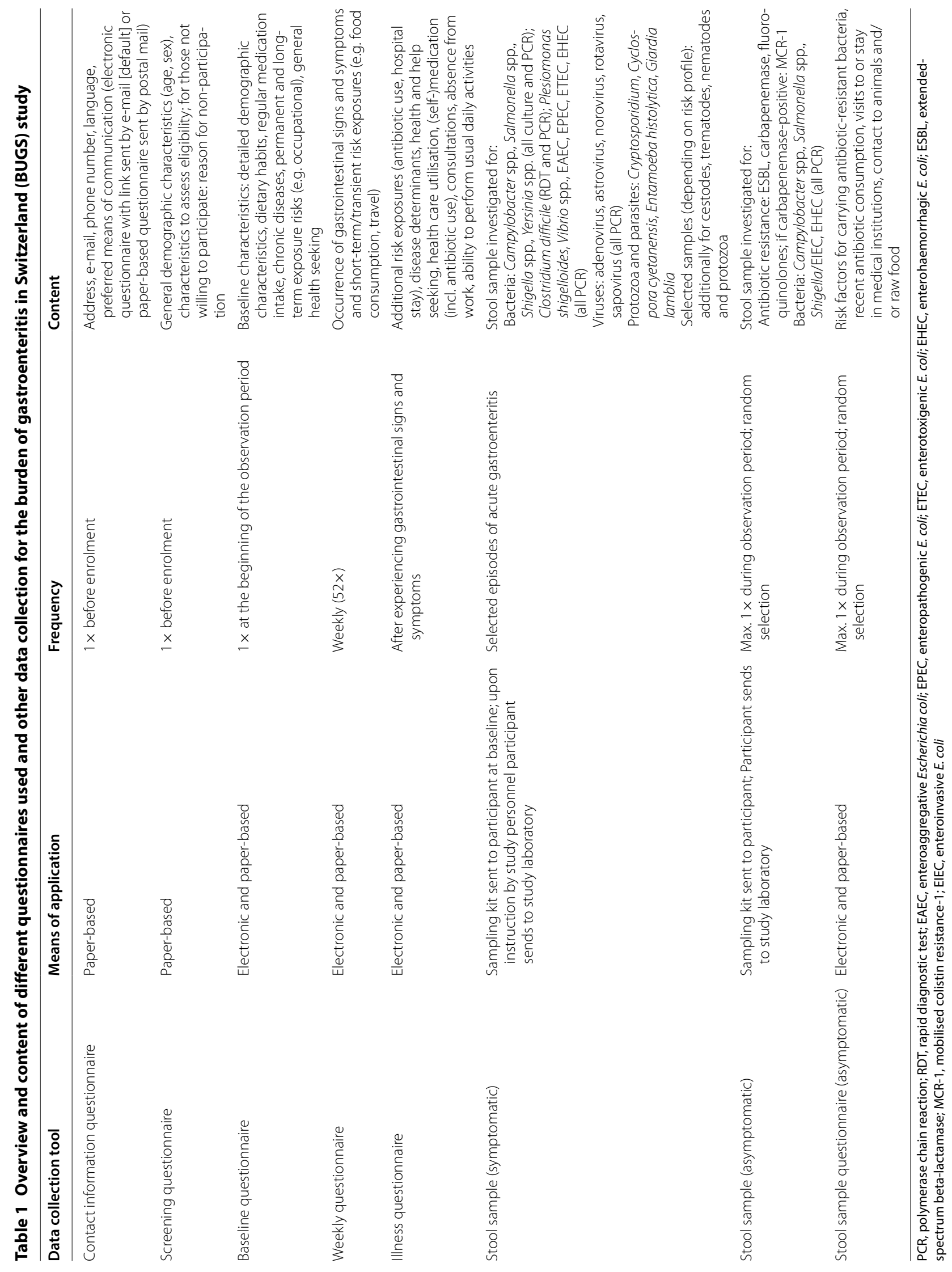




\section{BUGS observation period}
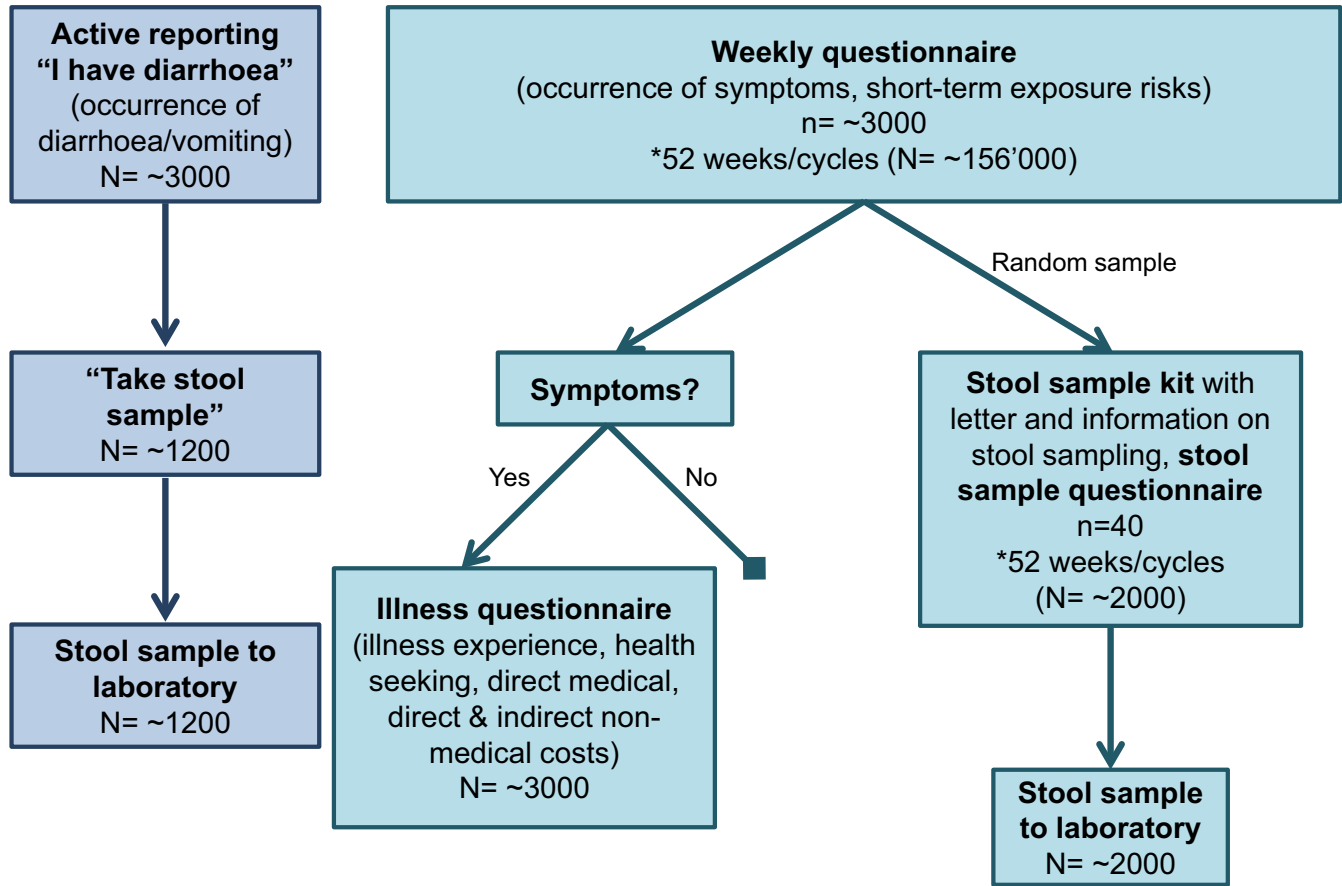

Fig. 2 Flowchart of cohort observation period for the burden of gastroenteritis in Switzerland (BUGS) study

e-mail or SMS (during the day, including weekends) (Figs. 2 and 3). This "active reporting system" is used to select AG episodes for microbiological investigation based on a pre-defined algorithm considering moderate and severe cases of illness as defined by Riddle et al. [19]. In case the study subject's AG episode is selected, the study team will advise him/her to use the stool sampling kit received at the beginning of the study and send a stool sample to the study laboratory as fast as possible. After sending in a stool sample, the participant's stool sampling kit will be replaced. The list of diagnostic tests performed on stool samples from symptomatic participants is provided in Table 1.

Each week, a pre-defined number of randomly selected participants (see sample size calculation) will receive a stool sampling kit to send in a stool sample immediately. Those stool samples will be tested for selected pathogenic bacteria and presence of certain antibiotic resistances with the aim to assess respective prevalences in the asymptomatic population (Table 1). The stool sample will be discarded if the participant reported diarrhoea with or without vomiting in the 4 weeks preceding sampling. Sampling is conducted weekly throughout the observation period to account for potential seasonal differences in prevalence. Each participant is selected at most once for "asymptomatic sampling".

All questionnaires apart from the contact information and screening questionnaires will be available in electronic- and paper-format. By default, participants will receive an e-mail containing a personalised link to the electronic questionnaires. However, during recruitment, they may opt for paper-based questionnaires sent by postal mail. LimeSurvey, an open source software set-up on a secured server at our institution (Swiss TPH), will be used for electronic data collection. The software allows completion of the questionnaire in a standard internet browser using desktop or laptop computers, tablets or smartphones.

Electronic questionnaires will be programmed to require an answer to ensure completeness of the data collected but will include an option "I do not want to answer" or "not applicable" (where appropriate) in order to prevent a forced-choice bias.

Paper questionnaires are entered by study personnel at the study centre using a LimeSurvey data entry mask which is slightly adapted from the one used by participants (e.g. containing an additional field "no answer given" for each mandatory question). A sample of $10 \%$ of paper questionnaires is entered twice for data quality 


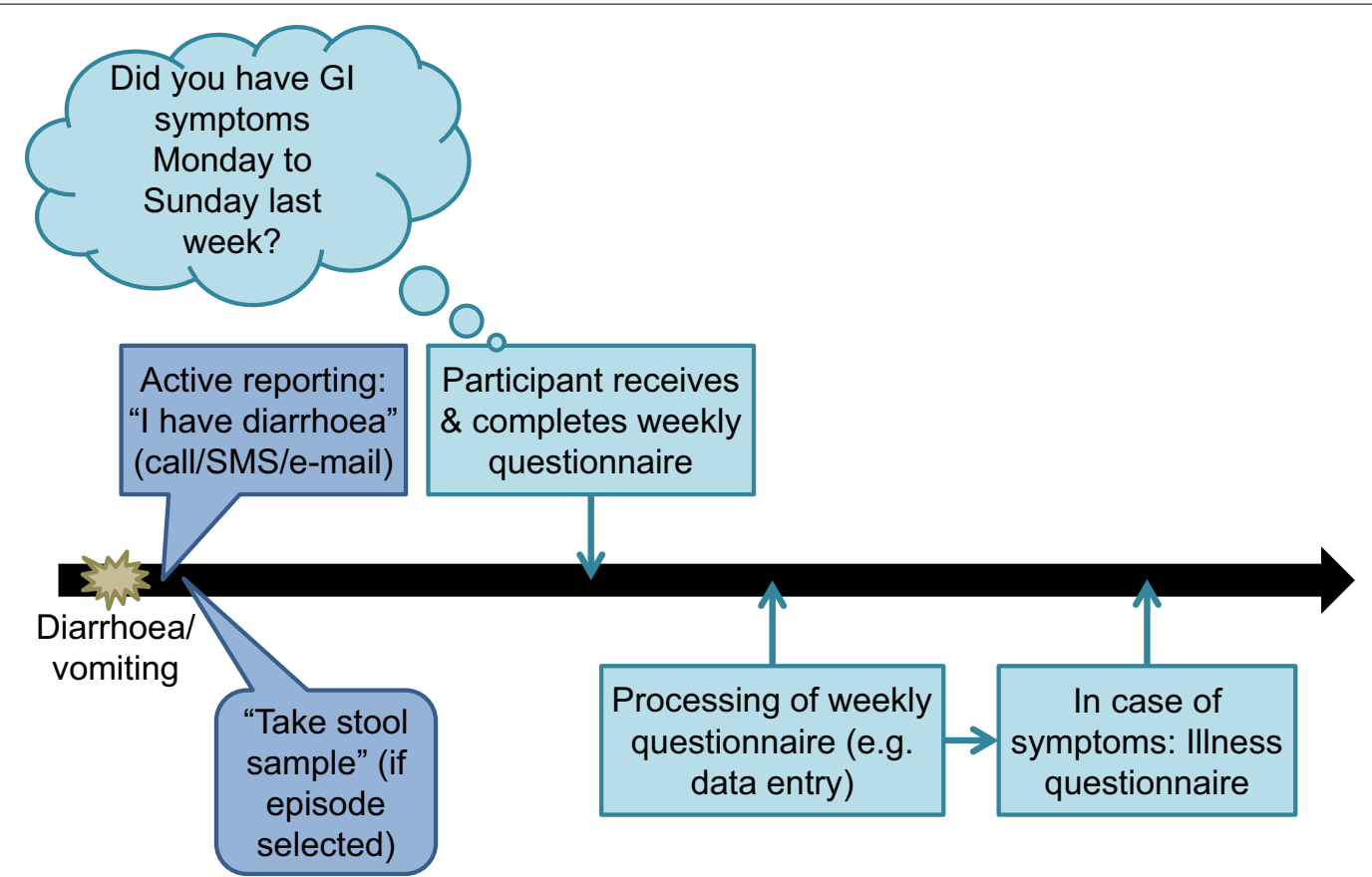

Fig. 3 Timeline from occurrence of symptoms until sending of illness questionnaire for the burden of gastroenteritis in Switzerland (BUGS) study. Gl, gastrointestinal

control. Discordant results are checked against paper originals. A full double entry is conducted for the "contact information questionnaire" as correct address details are crucial for successfully contacting participants.

Quality control of data from study laboratories (stool sample results) will be with the diagnostic laboratories, operating according to their standard operating procedures in their daily routine. The study team will only check plausibility of the data and values.

Electronic data are stored on secured network drives accessible only by the study team. Data on the network drive is backed-up regularly, according to institutional policy. Electronic data is stored in original file formats and in comma-separated values (csv) format, where appropriate, to ensure long-term accessibility.

\section{Sample size calculation}

We calculated the minimal sample size based on different parameter assumptions for comparisons between two distinct groups and for within-subject comparisons between two distinct periods. Underlying formulas and results are provided in Additional file 1.

A cohort size of 3000 individuals is envisaged to allow analysis of secondary outcomes and comparisons between groups and to assert enough power in case assumptions were too optimistic based on the different sample size calculations. We plan to start the observation period with a cohort size of 3500 individuals in order to achieve an average cohort size of 3000 after withdrawals and loss to follow-up during the 52 weeks study period. Assuming a participation rate of $25 \%$ (including loss of people not meeting the eligibility criteria) and a loss to follow-up of $20 \%, 15,000$ people will be contacted initially. For planning purposes, we assume an average cohort size of 3000 throughout the 1-year observation period.

The sample size (n) needed to reach a specific relative precision $(\varepsilon)$ for the microbiological outcomes in asymptomatic participants (prevalence of antibiotic resistances and selected pathogenic bacteria) was calculated using the following formula (based on [20]):

$$
n=\frac{1.96^{2} *(1-P)}{\varepsilon^{2} * P}
$$

For the prevalence of ESBL, a relative precision of $20 \%$ is envisaged at a $95 \%$ confidence level. Assuming a prevalence $(\mathrm{P})$ of $5-6 \%$ (based on [21, 22]), a sample size of $1505-1825$ is needed. Considering that the previous prevalence estimates are from groups of potentially higher prevalence than the general population (staff members of meat-processing companies with likely higher exposure risk; and primary care patients), we plan to investigate 2000 samples. Hence, every week 40 subjects are randomly selected. Given the very low prevalence of Carbapenemase-resistance (0.1\%; personal 
communication) and mcr-1 (not found in 1000 samples; personal communication) it is questionable whether these resistances will be found at all in our cohort. The prevalence of fluoroquinolone-resistance in E. coli has not yet been investigated in the asymptomatic Swiss population. Fluoroquinolone resistance prevalence in E. coli is at around $20 \%$ based on resistance data generated during routine medical care [23]. Hence, using the same sample size as for ESBL $(\mathrm{N}=2000)$ should allow for an estimate with a relative precision of at least $20 \%$ even if the prevalence in the general Swiss population is somewhat lower than $20 \%$.

The presence of pathogenic bacteria (EHEC, Campylobacter spp., Salmonella spp., and Shigella spp.) can only be investigated in 1600 samples of asymptomatic participants (of 2000 collected) due to financial constraints. For this investigation, the main interest is on EHEC prevalence in asymptomatic people. Considering that this prevalence is expected at around 7-10\% (personal communication) a sample size of 1600 will still allow for a relative precision of $15-18 \%$.

\section{Approach to analysis Definition of AG disease episode}

The primary outcome of our study is presence or absence of an AG episode and the incidence of AG in the general population. For this purpose, an episode of AG is classified according to a modified version of the definition suggested by Majowicz et al. [24]: a case of AG is an individual with $\geq 3$ loose stools in $24 \mathrm{~h}$, with or without vomiting, but excluding those (a) with cancer of the bowel, irritable bowel syndrome, Crohn's disease, ulcerative colitis, cystic fibrosis, coeliac disease, or another chronic illness with symptoms of diarrhoea, or (b) who report their symptoms were due to drugs, alcohol, or pregnancy. An episode is defined to begin on the first day and end on the last day of reported diarrhoea and/or vomiting, followed by a diarrhoea- and vomiting-free period of 3 days [25].

\section{Statistical analysis}

Data cleaning and analysis will be conducted with the statistical software Stata and/or R. All steps performed to clean and analyse the data are documented in scripts (R) or do-files (Stata).

The cohort will be characterised in terms of demographic characteristics, health status, dietary habits and permanent or long-term risk exposures as reported at baseline. Similarly, cohort participants lost to follow-up will be compared to those remaining in the cohort.

The primary outcome defined at the level of personweek is presence or absence of an AG episode. Risk factors for experiencing an episode of AG will be determined using multivariable mixed logistic regression analyses with the individual included as a random effect. A basic multivariable model including the biologically most plausible variables, region and season will be defined a priori. Generalised estimating equation (GEE) models will be considered in case of convergence issues of the mixed logistic regression models.

Secondary analyses will include calculating the incidence of AG as the number of AG episodes per person-year under observation and the incidence of gastrointestinal signs and symptoms (including episodes not fulfilling the case definition of AG). Furthermore, transient risk factors for experiencing an episode of AG will be explored in uni- and multivariable mixed logistic regression models, including the individual as random effect. Exposure information from the week preceding the AG episode will be used. Determinants for presenting to the health system (pharmacies, primary care, specialists or hospitals) in case of AG are also investigated, again using uni- and multivariable mixed logistic regression models (with the individual as random effect). Predictor variables include signs and symptoms, and perceived severity of AG, demographic characteristics (age, sex, occupational status), co-morbidity, and type of health insurance. Additionally, the socio-economic impact such as absence from work, inability to perform usual daily activities and the need for care by family members or friends related to AG disease episodes will be described.

Results from stool sample investigations from symptomatic participants will be used to estimate pathogenspecific incidence rates of AG.

Finally, the prevalences of bacteria harbouring ESBL-, carbapenem- and fluoroquinolone-resistance, and of EHEC, Campylobacter, Salmonella and Shigella in the asymptomatic population are calculated.

\section{Ethical considerations}

Health-related personal data and stool samples from participants are collected for this study. Therefore, the study is subject to the Federal Act on Research on Human Beings and requires ethical approval. Approval will be sought from the responsible local ethical committee(s) for a non-clinical trial study with minimal risks. All participants will be asked for written informed consent before enrolment. The study will be conducted according to the principles of Good Epidemiological Practice [26] and the Declaration of Helsinki [27].

Participants will be advised to seek health care as they would do without participating in this study. Similarly, it will be emphasised that investigation of stool samples in the framework of this study does not replace stool sample investigation potentially initiated by their health care provider. Results of stool investigations of 
symptomatic participants conducted as part of this study are obtained with a time delay due to study logistics. Hence, microbiological results will be known to the study team too late to affect treatment considering the short disease duration of most AG episodes and, therefore, will not be communicated to participants or their physicians.

Participants are informed if antibiotic resistances are identified in their stool samples during an asymptomatic period and advised to inform their physician in case of illness.

\section{Operational issues}

\section{Data confidentiality and personalisation}

Ideally, questionnaires should be anonymous as soon as they contain health-related personal information. However, we must be able to link the different questionnaires completed by each study participant. To minimise the risk that unauthorised people are able to link the participants' names to their questionnaire data, we plan to implement several measures: each participant is given a person identification code ("person ID") as well as a questionnaire identification code ("questionnaire ID"). The person ID is used in all files/data sets containing personal information. The questionnaire ID is used in all files/data sets containing information obtained from questionnaires or from laboratory testing. The key file, linking the person ID and the questionnaire ID will be password protected and stored separately from the other data sets. Access to this key file will be limited to the principal investigator, the study coordinator and an additional person (substitute of the study coordinator). For paper-based questionnaires, both codes (person ID and questionnaire ID) are printed on the empty questionnaires. However, as soon as receipt of the completed questionnaires is registered in the study centre, the person ID will be removed (cut off). In order to avoid that both codes are easily visible and recognised as such by unauthorised persons, one of the codes (the person ID) is printed in directly readable format (Arabic letters and numerals) and the other code is printed as a QR- or bar-code. Also, the two codes are not labelled with "person ID" or "questionnaire ID". For electronic questionnaires only the "questionnaire ID" will be used and no names are stored in LimeSurvey (where questionnaire data is entered and stored during the entire data collection phase). In order to complete the questionnaire, participants will receive an e-mail including a link to the questionnaire containing the questionnaire ID.

\section{Active reporting system needed}

For investigating the aetiology of AG, stool samples need to be obtained quickly after disease onset as AG is usually a short, self-limiting disease. Therefore, the information on signs and symptoms obtained through the weekly questionnaire is too much delayed to be the basis for selection of episodes for aetiological investigation (especially in those completing the paper-based questionnaires; Fig. 3). We expect reporting delays of 1-13 days in those completing electronic questionnaires and of at least 3-10 days (best case scenario) for paper-based questionnaires which will severely impact the likelihood to detect pathogens. To address this limitation, we plan to set up an "active reporting system" for participants (see "Data collection and management"). These parallel ways of collecting data on diarrhoea and vomiting have advantages and disadvantages: on the one hand, we can compare reporting completeness of the two methods. On the other hand, participants are required to actively think of the study when experiencing signs and symptoms, and have to report their symptoms twice-potentially increasing reporting fatigue and reporting bias due to sensitisation. Further, the active reporting system requires that study personnel being able to communicate in all three study languages is on call every day, including weekends and public holidays.

\section{Limitations}

Our study will be subject to limitations. Participation bias is likely to occur. Considering the rather long observation period (1 year with weekly follow-ups), a certain amount of participants will be lost to follow-up or withdraw from the study; these participants might differ from those completing the entire follow-up period. Similarly, reporting fatigue might occur, especially in those experiencing more than one episode of AG. However, all those biases and limitations are unavoidable in cohort studies and their mitigation is difficult. To address reporting fatigue, we consider establishing a project newsletter to give participants feedback about interim results of the study and additional information related to the topic to highlight the importance of their contribution to research. Inverse probability weighting will be considered if characteristics of our final cohort differ from those of the general population.

Furthermore, compliance in actively reporting signs and symptoms as well as in providing stool samples is crucial. Therefore, its importance will be emphasised repeatedly to study participants. We suspect that compliance might be associated with the (perceived) severity of the disease episode. However, we can only try to assess this bias at the end of the study by comparing data from active reporting/stool sampling and data from weekly questionnaires.

Recall bias and telescoping have been described as major challenges in studies on AG. We believe that this 
bias might not be a notable problem in our study considering the rather short recall period of 1 week.

Participants travelling will still receive the electronic questionnaire but those completing paper-based questionnaires will be able to complete the questionnaire only once they returned. This might lead to different compliance and recall bias in those two groups. On the other hand, it provides an opportunity to assess potential differences in results between the two methods.

\section{Additional file}

Additional file 1. Sample size calculations and underlying formulas for the BUGS study. Sample size calculations for the burden of gastroenteritis in Switzerland (BUGS) study based on different parameter assumptions, including derivation of underlying formulas.

\section{Abbreviations}

AG: acute gastroenteritis; BUGS: burden of gastroenteritis in Switzerland [study]; EAEC: enteroaggregative Escherichia coli; EHEC: enterohaemorrhagic Escherichia coli; EIEC: enteroinvasive Escherichia coli; EPEC: enteropathogenic Escherichia coli; ESBL: extended-spectrum beta-lactamase; ETEC: enterotoxigenic Escherichia coli; FOPH: Federal Office of Public Health; mcr: mobilised colistin resistance; NNSID: National Notification System for Infectious Diseases; PCR: polymerase chain reaction; RDT: rapid diagnostic test.

\section{Authors' contributions}

DM and CS conceptualised the study and wrote the manuscript. Both authors read and approved the final manuscript.

\section{Author details}

${ }^{1}$ Swiss Tropical and Public Health Institute, Basel, Switzerland. ${ }^{2}$ University of Basel, Basel, Switzerland.

\section{Acknowledgements}

The authors thank Philipp J. Bless, Sören Becker and Roger Stephan for providing important inputs to earlier versions of this research proposal. Andreas Neumayr's and Esther Künzli's insights provided and their recommendations in dealing with issues related to medical practice are very much appreciated. The statistical advice provided by Jan Hattendorf and statistical advice and feedback to the manuscript by Christian Schindler are gratefully acknowledged.

\section{Competing interests}

The authors declare that they have no competing interests.

\section{Availability of data and materials}

Data sharing not applicable to this article as datasets were not yet generated for the presented study.

\section{Consent for publication}

Not applicable.

\section{Ethics approval and consent to participate}

Approval for this study will be sought from the responsible local ethical committee(s) for a non-clinical trial study with minimal risks. All participants will be asked for written informed consent. The study will be conducted according to the principles of Good Epidemiological Practice and the Declaration of Helsinki.

\section{Funding}

This study is funded by the Federal Office of Public Health (FOPH; Bern, Switzerland) and the Federal Food Safety and Veterinary Office (FSVO; Bern, Switzerland). The FOPH and the FSVO expressed specific research needs which this study should answer but did not have any role in the design of the study and in writing the manuscript.

\section{Publisher's Note}

Springer Nature remains neutral with regard to jurisdictional claims in published maps and institutional affiliations.

Received: 22 August 2018 Accepted: 9 November 2018

Published online: 16 November 2018

\section{References}

1. Tam CC, Rodrigues LC, Viviani L, Dodds JP, Evans MR, et al. Longitudinal study of infectious intestinal disease in the UK (IID2 study): incidence in the community and presenting to general practice. Gut. 2012;61:69-77.

2. Scallan E, Fitzgerald M, Collins C, Crowley D, Daly L, et al. Acute gastroenteritis in northern Ireland and the Republic of Ireland: a telephone survey. Commun Dis Public Health. 2004;7:61-7.

3. Wilking $H$, Spitznagel $H$, Werber $D$, Lange $C$, Jansen $A$, et al. Acute gastrointestinal illness in adults in Germany: a population-based telephone survey. Epidemiol Infect. 2013;141:2365-75.

4. Doorduyn Y, Van Pelt W, Havelaar AH. The burden of infectious intestinal disease (IID) in the community: a survey of self-reported IID in The Netherlands. Epidemiol Infect. 2012;140:1185-92.

5. Scavia G, Baldinelli F, Busani L, Caprioli A. The burden of self-reported acute gastrointestinal illness in Italy: a retrospective survey, 2008-2009. Epidemiol Infect. 2012;140:1193-206.

6. Kuusi M, Aavitsland P, Gondrosen B, Kapperud G. Incidence of gastroenteritis in Norway—a population-based survey. Epidemiol Infect. 2003;131:591-7.

7. Müller L, Korsgaard H, Ethelberg S. Burden of acute gastrointestinal illness in Denmark 2009: a population-based telephone survey. Epidemiol Infect. 2012;140:290-8.

8. Hansdotter Fl, Magnusson M, Kuhlmann-Berenzon S, Hulth A, Sundstrom $\mathrm{K}$, et al. The incidence of acute gastrointestinal illness in Sweden. Scand J Public Health. 2015;43:540-7.

9. Edelstein M, Merk H, Deogan C, Carnahan A, Wallensten A. Quantifying the incidence and cost of acute gastrointestinal illness in Sweden, 2013-2014. Epidemiol Infect. 2016;144:2831-9.

10. Viviani L, van der Es M, Irvine L, Tam CC, Rodrigues LC, et al. Estimating the incidence of acute infectious intestinal disease in the community in the UK: a retrospective telephone survey. PLOS ONE. 2016;1 1:e146171.

11. Baumann-Popczyk A, Sadkowska-Todys M, Rogalska J, Stefanoff P. Incidence of self-reported acute gastrointestinal infections in the community in Poland: a population-based study. Epidemiol Infect. 2012;140:1173-84.

12. Van Cauteren D, De Valk H, Vaux S, Le Strat Y, Vaillant V. Burden of acute gastroenteritis and healthcare-seeking behaviour in France: a populationbased study. Epidemiol Infect. 2012;140:697-705.

13. Gauci C, Gilles H, Mamo J, Ruggieri F-M, Di Bartolo I, et al. The aetiology of infectious intestinal disease in the community in Malta. Malta Med J. 2010;22:6-12.

14. Grilc E, Sočan M. Population based self-reported acute gastrointestinal infection in Slovenia: multiplier study. Slovenian J Public Health. 2014:53:125-32.

15. Klem F, Wadhwa A, Prokop $\sqcup$, Sundt WJ, Farrugia G, et al. Prevalence, risk factors, and outcomes of irritable bowel syndrome after infectious enteritis: a systematic review and meta-analysis. Gastroenterology. 2017;152(1042-1054):e1041.

16. $\mathrm{Ng}$ QX, Soh AYS, Loke W, Lim DY, Yeo WS. The role of inflammation in irritable bowel syndrome (IBS). J Inflamm Res. 2018;11:345-9.

17. Gibbons CL, Mangen MJJ, Plass D, Havelaar AH, Brooke RJ, et al. Measuring underreporting and under-ascertainment in infectious disease datasets: a comparison of methods. BMC Public Health. 2014;14:147.

18. Schmutz C, Bless PJ, Mäusezahl D, Jost M, Mäusezahl-Feuz M, et al. Acute gastroenteritis in primary care: a longitudinal study in the Swiss Sentinel Surveillance Network, Sentinella. Infection. 2017;45:811-24.

19. Riddle MS, DuPont HL, Connor BA. ACG clinical guideline: diagnosis, treatment, and prevention of acute diarrheal infections in adults. Am J Gastroenterol. 2016;111:602. 
20. Lwanga SK, Lemeshow S. Sample size determination in health studies. A practical manual. Geneva: World Health Organization; 1991.

21. Geser N, Stephan R, Korczak BM, Beutin L, Hächler H. Molecular identification of extended-spectrum-beta-lactamase genes from Enterobacteriaceae isolated from healthy human carriers in Switzerland. Antimicrob Agents Chemother. 2012;56:1609-12.

22. Nüesch-Inderbinen MT, Abgottspon H, Zurfluh K, Nüesch HJ, Stephan $\mathrm{R}$, et al. Cross-sectional study on fecal carriage of Enterobacteriaceae with resistance to extended-spectrum cephalosporins in primary care patients. Microbial Drug Resistance. 2013;19:362-9.

23. Swiss Centre for Antibiotic Resistance. Antibiotic resistance data. http:// anresis.ch/index.php/Interactive-database.html. Accessed 21 Feb 2018.
24. Majowicz SE, Hall G, Scallan E, Adak GK, Gauci C, et al. A common, symptom-based case definition for gastroenteritis. Epidemiol Infect. 2008;136:886-94.

25. Morris SS, Cousens SN, Lanata CF, Kirkwood BR. Diarrhoea-defining the episode. Int J Epidemiol. 1994;23:617-23.

26. Altpeter E, Burnand B, Capkun G, Carrel R, Cerutti B, et al. Essentials of good epidemiological practice. Soz Praventivmed. 2005;50:12-27.

27. World Medical Association. World Medical Association Declaration of Helsinki: ethical principles for medical research involving human subjects. JAMA. 2013;310:2191-4.
Ready to submit your research? Choose BMC and benefit from:

- fast, convenient online submission

- thorough peer review by experienced researchers in your field

- rapid publication on acceptance

- support for research data, including large and complex data types

- gold Open Access which fosters wider collaboration and increased citations

- maximum visibility for your research: over $100 \mathrm{M}$ website views per year

At BMC, research is always in progress.

Learn more biomedcentral.com/submissions 